\title{
Diseño de un prototipo para el control de lámparas empleando tecnología inalámbrica
}

\author{
Ma. Daniela Ronquillo Pilamunga / César Martín Moreno
}

\begin{abstract}
RESUMEN
Este trabajo consiste en el diseño de un prototipo para el control de lámparas como una propuesta para el auditorio de la Fundación Leonidas Ortega Moreira (FLOM). El prototipo busca facilitar el uso correcto de las lámparas como fluorescentes y luces incandescentes distribuidas dentro del auditorio, además de reducir el tiempo para el control de los mismos. El prototipo está elaborado a pequeña escala utilizando módulos leds para simular las lámparas del auditorio. Además emplea tecnología Bluetooth en conjunto con un dispositivo móvil con sistema operativo Android como herramienta de control a través de una aplicación para manejar los estados de encendido y apagado de cada dispositivo lumínico del auditorio. Adicional a esto se ha implementado funcionalidades como temporizar, controlar el nivel de intensidad, encender y apagar todas las lámparas con un toque táctil, todo esto desde una tablet con la aplicación Android. Finalmente se realizó la instalación de las lámparas en una maqueta y se llevaron a cabo las pruebas del prototipo: prueba de conexión, prueba de encendido y apagado, prueba de temporización y prueba de control de intensidad; las cuales dieron resultados satisfactorios. Asimismo se realizó una prueba de tiempo de respuesta del prototipo la cual cumplió con las expectativas permitiendo lograr con los objetivos establecidos: garantizar un mejor control y optimizar el tiempo del uso de las lámparas.
\end{abstract}

\section{PALABRAS CLAVE}

Bluetooth, Android, microcontrolador, domótica, control.

\begin{abstract}
This paper refers to the design of a prototype of lighting control devices as an alternative for the auditorium of the Leonidas Ortega Moreira Foundation (FLOM). The prototype aims to facilitate the proper use of lighting devices such as fluorescent and incandescent lights distributed within the auditorium, and reduce the time to control them. Moreover, this apparatus was made and tested in small scale using led modules to simulate the auditorium lamps. Besides, it uses Bluetooth technology in addition to the Android operating system for a mobile device, as a control tool through an application in order to handle the $\mathrm{ON}$ and $\mathrm{OFF}$ status of each light device in the auditorium. Further to this, there are some functionality implemented, for example: timing, control of the level of intensity, and control switch for the on and off function in all lighting devices using a tactile touch device, all possible using the Android application. Finally, the prototype was tested on a scale model. Those tests include: connection, on and off functions, timing and intensity control, which satisfactory results. Furthermore, a response time test of the prototype met the expectations. This allows to fulfill the objectives proposed at the beginning, ensuring a better control and to optimize the use in lighting devices.
\end{abstract}

\section{KEYWORDS}

Bluetooth, Android, microcontroller, domotic, control. 


\section{Introducción}

La Fundación Leonidas Ortega Moreira (FLOM) es una institución cuya misión es: contribuir en la educación del país. Siempre se encuentra en constante labor entregando becas, realizando capacitaciones a docentes, becarios y público en general, participando activamente en programas relacionados con la educación.

Algunos de estos programas y capacitaciones se realizan en el auditorio de la institución, llegando a convertirse en un espacio importante ya que es sede de varios eventos educativos cada año. Para conocer la situación actual del uso de las lámparas del auditorio se realizaron encuestas y entrevistas al personal de la FLOM, mediante las cuales se pudo apreciar que existian inconvenientes y retrasos al momento de controlarlos, uno de los inconvenientes es que el control vigente de las lámparas requiere imperativamente que una persona esté de pie junto a los controles durante todo un evento además esta persona debe conocer el uso correcto de cada interruptor.

Por otro lado, la necesidad de interconectar dispositivos electrónicos de forma inalámbrica ha llevado a la creación de tecnologías que permiten lograr este objetivo, de esta manera se prescinde de medios como el cable (Molisch, 2010).

De igual forma, el ser humano en su afán de automatizar aparatos eléctricos ya sea dentro de una vivienda, edificio o industria ha buscado plantear soluciones con la domótica y la inmótica (Martín, 2009).

Dentro del ámbito de la domótica existen diferentes tecnologías inalámbricas como: WiFi, Bluetooth, ZigBee (Junestrand, Passaret, \& Vázquez, 2005). Cada tecnología inalámbrica tiene características que las convierten en soluciones optimas dependiendo del uso que se le quiera dar, e incluso en los sistemas domóticos algunas tecnologías pueden ser complementos de otras.

El estándar WiFi a pesar de su velocidad de transmisión de 50 Mbps y su gran alcance de 100 metros en comparación con otras tecnologías genera un consumo energético más elevado con respecto a Bluetooth y ZigBee (SCD).

En cambio las tecnologías inalámbricas Bluetooth y ZigBee destacan por sus buenas cualidades en cuanto se refiere a automatización y control (Bluetooth-SIG (2013); ZigBee Alliance (2013)).

No obstante para este prototipo la tecnología Bluetooth presenta más bondades para el desarrollo y facilidades de acceso en múltiples dispositivos móviles, es por esta razón que se escogió esta tecnología. El uso de la tecnología de comunicación Bluetooth se ha extendido a múltiples aplicaciones y actualmente tiene presencia en gran cantidad de dispositivos móviles, equipos de computación, entre otros (Bluetooth SIG, 2013).

Los dispositivos móviles forman parte de las actividades diarias ya que puede tener múltiplos usos. Estos dispositivos cuentan con sistemas operativos propios, siendo el más común Android (Gironés, 2012).

Android es el sistema operativo móvil que ha conseguido mantenerse como la plataforma más utilizada, esto se lo puede comprobar revisando las estadísticas concernientes a las cuotas de mercado de los sistemas operativos móviles hasta el 2014.

Según un estudio realizado por IDC (2014): En el primer trimestre del 2014, Android mantiene una cuota de mercado del $81.1 \%$, por un $15.2 \%$ de iOS, un $2.7 \%$ de Windows Phone, un $0.5 \%$ de BlackBerry y un $0.6 \%$ de otros. 
Android se ha convertido en uno de los principales sistemas operativos empleados a nivel mundial. La facilidad de desarrollo $y$ publicación de aplicaciones para el mismo, le han dado este realce dentro del mercado. Android también brinda soporte al estándar Bluetooth y es por este motivo que se convierte en un aliado para el desarrollo de aplicaciones que empleen este estándar.

El objetivo de este trabajo es elaborar el diseño de un prototipo que permita el control de las lámparas para ello se utilizará la tecnología Bluetooth para la comunicación y como herramienta de control una aplicación Android. De manera que se pueda mejorar el control de las lámparas. El uso del prototipo propuesto puede extenderse a otros espacios y aplicaciones, como por ejemplo controlar más dispositivos en una vivienda o en una oficina.

\section{Marco Teórico}

El desarrollo tecnológico en que se desenvuelve nuestro mundo hace posible el surgimiento de nuevos dispositivos, aplicaciones, invenciones $\mathrm{y}$ conocimientos que ayudan a mejorar no solo la calidad de vida del ser humano, sino que también favorecen a su crecimiento en varios aspectos (empresarial, intelectual, personal, etc.).

Las telecomunicaciones han evolucionado en rendimiento, capacidad y con la finalidad de brindarle comodidad al usuario. Según José Huidobro experto en Telecomunicaciones señala que los avances científicos en especial dentro del área de las telecomunicaciones "... ban cambiado de manera radical el comportamiento del ser bumano, dándole la posibilidad de comunicarse, de muy diversas maneras y casi de manera instantánea, con casi cualquier otra persona o máquina de este planeta" (Huidobro, 2011, pág. IX).
De estos avances, en el área de las telecomunicaciones han surgido varios estándares de transmisión inalámbricos, diseñados especialmente para evitar el cableado de dispositivos los cuales previamente era imposible interconectarse por otro medio que no fuesen cables, como por ejemplo el estándar Bluetooth (IEEE 802.15.1).

Huidobro (2011) señala lo siguiente:

Bhtuetooth es el nombre cominn de la especificación industrial IEEE 802.15.1, que define In estándar global de commnicación inalambrica que posibilita la transmisión de voz $y^{\prime}$ datos entre diferentes dispositivos mediante mn enlace por radiofrecuencia segura, globalmente y sin licencia de corto rango. (pág. 150)

La tecnología Bluetooth está integrada actualmente en móviles, computadores, cámaras, impresoras, equipos para estudios deportivos y gran variedad de hardware; asi de esta manera ha logrado adquirir una gran importancia en el mundo de las telecomunicaciones.

De la misma manera, los teléfonos móviles, han revolucionado la forma de comunicarse. "Un teléfono móvil siempre está en el bolsillo del usuario, esto le convierte en el nuevo ordenador personal del siglo XXI" (Gironés, 2012).

Actualmente, los dispositivos conocidos como teléfonos inteligentes cuentan con una gran capacidad de procesamiento y almacenamiento lo cual brinda al usuario una experiencia agradable. Estos dispositivos cuentan con su propio sistema operativo y una gran cantidad de aplicaciones.

De acuerdo a un estudio realizado en el primer cuatrimestre del 2013 por Millennial Media, una compañía dedicada a la publicidad móvil y plataforma de datos, señala que la mayoría de los teléfonos inteligentes usan Android como sistema operativo. El $52 \%$ 
de los smartphones tienen sistema operativo Android, frente al $39 \%$ que tiene iOS y un $7 \%$ RIM, el sistema operativo de BlackBerry (Millennial Media, 2013).

Lee (2011) indica lo siguiente:

Android es un sistema operativo para dispositivos móviles que se basa en una versión modificada de Linux. Fue desarrollado originalmente por Android, Inc. En el 2005, Google, como parte de su estrategia para entrar en el mercado de dispositivos móviles, adquirió Android y se bizo cargo de su trabajo de desarrollo (así como de su equipo de desarrollo). (p. 2)

Este sistema operativo para dispositivos móviles permite usar su código fuente de manera gratuita a todos los desarrolladores a nivel mundial. Desde el 2009 el equipo de Google liberó la plataforma de desarrollo para Android ADT (Android Development Tools) con la finalidad de desarrollar aplicaciones móviles para el sistema operativo Android desde la plataforma de Eclipse (Android Developers, 2013).

En el 2010, Google pensando en hacer de la programación un área más accesible decide brindar una solución y crea "App Inventor". "App Inventor es una herramienta visual drag-anddrop para la creación de aplicaciones móviles en la plataforma Android. Permite diseñar la interfaz de usuario (el aspecto visual) de una aplicación mediante una interfaz, gráfica de usuario basada en Web GUI" (Wolber, Abelson, Spertus, \& Looney, 2011). Actualmente, los servicios de App Inventor, son proporcionados por el Massachusetts Institute of Technology (MIT).

Mientras que en el 2013, Google anuncia en su conferencia anual a Android Studio como el entorno de desarrollo integrado (IDE) oficial para desarrollar aplicaciones Android.
La electrónica, las telecomunicaciones y la informática van de la mano, abriendo paso a varias soluciones que emplean estas áreas para el control y la automatización. Ante los grandes avances de las tecnologías expuestas anteriormente, se vuelve factible emplear estas tecnologías para desarrollar soluciones prácticas para el control de dispositivos a distancia.

\section{Elementos de hardware y software para el prototipo de control de las lámparas}

A grandes rasgos, el bosquejo del prototipo de control de las lámparas se encuentra conformado por software y hardware; ya que por un lado el software incluye un conjunto de herramientas de desarrollo que permitirán el diseño de una aplicación móvil de control y por otro lado, el hardware incluye todas las herramientas electrónicas como circuitos integrados, microcontroladores, entre otros componentes que permitirán construir el circuito electrónico necesario para controlar las lámparas. Para la selección del hardware y software es necesario detallar los requerimientos del prototipo.

\section{Requerimientos del prototipo}

El prototipo del control de las lámparas requiere de un microcontrolador que debe contar con: 8 salidas digitales que permiten manipular el encendido y apagado de las lámparas y 8 entradas digitales que permiten manipular las lámparas (encendido/apagado) manualmente a través de pulsadores, esto se debe a que son 8 juegos de lámparas; 2 salidas con modulación de ancho de pulso (PWM) que sirven para los dos juegos de dimmers.

La Interfaz USART (Tx-Rx) es necesaria para establecer una comunicación entre el controlador principal y el módulo inalámbrico, siendo el módulo inalámbrico el dispositivo que se enlazará con un dispositivo móvil que será controlado por un usuario. 
En la Figura 1 se detalla el diagrama de bloques del prototipo del control de lámparas, este diagrama es la parte de hardware del prototipo.

\section{Figura \# 1}

Diagrama de bloques del control de lámparas.

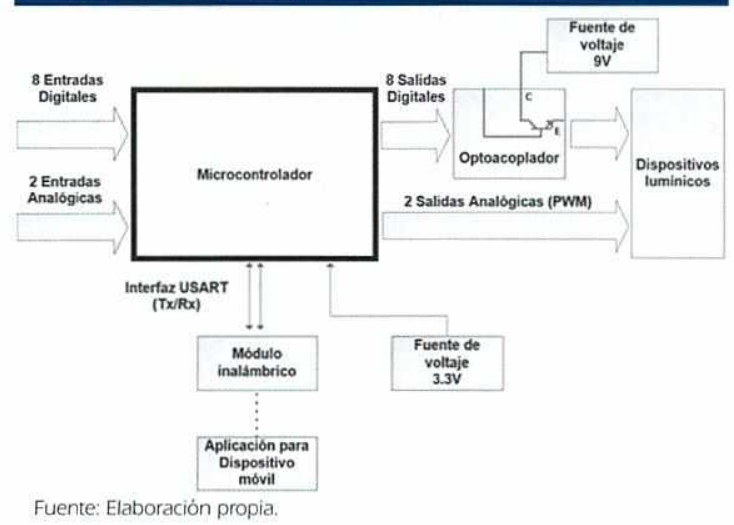

Teniendo en consideración el diagrama de bloques se realizará un análisis del módulo inalámbrico Bluetooth y un análisis del entorno de desarrollo integrado para la aplicación móvil con sistema operativo Android.

\section{Análisis del módulo}

\section{inalámbrico Bluetooth}

Bluetooth se encuentra presente en el mercado desde 1994. Desde ese año hasta la actualidad ha incorporado varias mejoras en cada versión desarrollada y a la par con este desarrollo, ha formado una alianza que reúnen a varias empresas importantes a nivel internacional para que puedan implementar y fortalecer la presencia de Bluetooth en los diferentes dispositivos que ofrecen en el mercado. Es esta una de las razones por las cuales, actualmente se puede encontrar esta funcionalidad estándar incorporada en los móviles, tablets, laptops, entre otros dispositivos, de manera que se pueda garantizar al usuario de un poderoso medio para interconectar sus dispositivos de manera rápida $\mathrm{y}$ sencilla.

\section{Módulos inalámbricos Bluetooth existentes en el mercado}

Un módulo inalámbrico Bluetooth es un componente de hardware que funciona con tecnología Bluetooth que permite el intercambio de datos y audio entre dispositivos que también cuenten con esta tecnología, reemplazando así al cableado (comunicación por cable). Un módulo inalámbrico Bluetooth es un componente que puede ser integrado dentro de un proyecto electrónico (Sequoia-Technology).

Existe una gran gama de módulos inalámbricos Bluetooth en el mercado con fabricantes reconocidos tales como: Bluegiga, Nordic Semiconductor, Texas Instruments. Estos fabricantes ofrecen un sin número de módulos inalámbricos Bluetooth, algunos son ofrecidos en kits para complementarlos con herramientas de desarrollo, siendo generalmente de costos elevados.

Por otro lado en el mundo de la electrónica son muy utilizados los módulos inalámbricos Bluetooth como el RN41, RN42, HC06, HC05 que son dispositivos económicos, con amplia disponibilidad comercial en el mercado.

\section{Comparación de los módulos}

\section{inalámbricos Bluetooth HC06 y RN41}

En la Tabla 1 se muestra la comparación entre los módulos inalámbricos Bluetooth RN41 y HC06.

Los módulos inalámbricos Bluetooth RN41 y HC06 soportan la versión $2.1+$ EDR. Operan en la frecuencia $2.4 \mathrm{GHz}$. Ambos tienen una sensibilidad de receptor de $-80 \mathrm{dBm}$ por lo contrario el RN41 y el HCO6 ofrecen una potencia de salida de $12 \mathrm{dBm}$ y $6 \mathrm{dBm}$ respectivamente. Ambos tienen interfaces: Transmisor-Receptor Asíncrono Universal UART, Bus de Serie Universal USB e Interfaz Periférica Serial SPI. 


\begin{tabular}{|c|c|c|}
\hline \multicolumn{3}{|c|}{$\begin{array}{l}\text { Caracteristicas de los módulos inalámbricos } \\
\text { Bluetooth RN41 y } \mathrm{HCO6}\end{array}$} \\
\hline Bluetooth & RN41 & $\mathrm{HCOG}$ \\
\hline Versión & Bluetooth $2.1+E D R$ & Bluetooth $2.1+$ EDR \\
\hline Soporte BLE & No & No \\
\hline Soporte BR/EDR & $\mathrm{Si}$ & $\mathrm{Si}$ \\
\hline Potencia de Transmisión / Tx Power & $12 \mathrm{dBm}$ & $6 \mathrm{dBm}$ \\
\hline Sensibilidad del receptor / Rx Sensitive & $-80 \mathrm{dBm}$ & $-80 \mathrm{dBm}$ \\
\hline Clase & 1 & 2 \\
\hline Rango (metros) & $100 \mathrm{~m}$ & $10 \mathrm{~m}$ \\
\hline Frecuencia & $2.412-2.484 \mathrm{GHz}$ & $2.4 \mathrm{GHz}$ \\
\hline Interfaz UART & Si & $\mathrm{Si}$ \\
\hline Interfaz USB & Si & Si \\
\hline Interfaz SPI & $\mathrm{Si}$ & Si \\
\hline Voltaje de Operación & $3.0-3.6 \mathrm{VDC}$ & $3.1-4.2 \mathrm{VDC}$ \\
\hline $\begin{array}{l}\text { Corriente durante establecimiento } \\
\text { de conexión }\end{array}$ & $30 \mathrm{~mA} \sim 50 \mathrm{~mA}$ & $30 \mathrm{~mA} \sim 40 \mathrm{~mA}$ \\
\hline Conexión establecida & $3 \mathrm{~mA}$ & $8 \mathrm{~mA}$ \\
\hline Corriente durante transmisión & $65 \mathrm{~mA}$ & $8 \mathrm{~mA}$ \\
\hline Modo descanso & $2.5 \mathrm{~mA}$ & Menor a $1 \mathrm{~mA}$ \\
\hline Dimensiones (mm) & $13.4 \times 25.8 \times 2$ & $27 \times 13 \times 2$ \\
\hline Costo (incluido IVA) & $\$ 50.40$ & $\$ 16.80$ \\
\hline
\end{tabular}

El módulo inalámbrico Bluetooth $\mathrm{HCO}$ tiene un bajo consumo de energía en comparación con el módulo inalámbrico Bluetooth RN41, y esto se debe a que el módulo inalámbrico Bluetooth RN41 requiere de una mayor potencia para alcanzar la cobertura de $100 \mathrm{~m}$ frente a los $10 \mathrm{~m}$ de cobertura del módulo inalámbrico Bluetooth HC06.

Ambos módulos inalámbricos Bluetooth cuentan con el perfil SPP (Serial Port Profile). Este perfil permite enviar o recibir datos TTL mediante la tecnología Bluetooth sin necesidad de conectar un cable serie a un ordenador. En el caso del módulo inalámbrico Bluetooth RN41, el perfil SPP trabaja a una velocidad de datos de $240 \mathrm{Kbps}$ cuando está configurado en modo esclavo y $300 \mathrm{Kbps}$ cuando está configurado en modo maestro. En el caso del módulo inalámbrico Bluetooth HCO6 también cuenta con el perfil SPP ya que fue diseñado para comunicaciones seriales, pero permite solo la configuración en modo esclavo.
Es importante mencionar que el módulo inalámbrico Bluetooth que se seleccione para el prototipo debe poseer: el perfil SPP, debe estar configurado en modo esclavo para que se pueda conectar ya sea a una computadora o a un dispositivo móvil como es el caso del prototipo a diseñarse $\mathrm{y}$ debe contar con interfaces UART. Ambos módulos cumplen con los tres primeros parámetros a excepción de la clase Bluetooth, ya que el módulo inalámbrico Bluetooth RN41 es de clase 1 y el módulo inalámbrico Bluetooth HCO6 es de clase 2. Trabajar con un módulo inalámbrico Bluetooth RN41 dará mayor cobertura en el auditorio pero por tratarse de un prototipo se ha decidido trabajar con el módulo inalámbrico Bluetooth HC06.

En un futuro si se desease optar por otro módulo Bluetooth esto implicaría cambios en la conexión de los pines del módulo basado en la hoja de datos del fabricante. De ser este el caso en la aplicación Android se debe colocar la dirección MAC del módulo Bluetooth al que se va a conectar, lo que significa cambios a nivel de software.

Análisis de los entornos de desarrollo integrado para el desarrollo de aplicaciones móviles con sistema operativo

\section{Android}

La selección de la IDE (Integrated Development Environment) es muy importante para el desarrollo de la aplicación, ya que la IDE permitirá la programación de la aplicación de una manera sencilla gracias a sus 
características y aportes para la escritura de código. Tal como: asistente de código, revisión de sintaxis, detección de errores. A continuación en la Tabla 2 se detalla una comparación de las principales IDEs que permiten desarrollar aplicaciones móviles con sistema operativo Android. se, como Android Studio, NetBeans y MIT App Inventor cuentan con depuración de código. Así mismo la detección de errores muestra todas las advertencias, errores y problemas que existan dentro de un proyecto. Tanto Eclipse, como Android Studio, NetBeans y MIT App Inventor cuentan con detección de errores.

\begin{tabular}{|c|c|c|c|c|}
\hline \multicolumn{5}{|c|}{$\begin{array}{l}\text { Comparación de las IDE para desarrollo de } \\
\text { aplicaciones Android }\end{array}$} \\
\hline Caracteristicas & Eclipse & $\begin{array}{l}\text { Android } \\
\text { Studio }\end{array}$ & $\begin{array}{l}\text { MIT App } \\
\text { Inventor }\end{array}$ & NetBeans \\
\hline Versión & $\begin{array}{l}\text { Eclipse Juno } \\
4.2\end{array}$ & $\begin{array}{l}\text { Versión Beta } \\
0.2 \\
\end{array}$ & Versión Beta & $\begin{array}{l}\text { NetBeans } \\
\text { IDE } 7.4\end{array}$ \\
\hline Asistente de código & $\mathrm{Si}$ & $\mathrm{Si}$ & No & $\mathrm{Si}$ \\
\hline Asistente de código & Si & $\mathrm{Si}$ & No & Si \\
\hline $\begin{array}{l}\text { Revisión de sintaxis } \\
\text { en tiempo real }\end{array}$ & Si & Si & $\mathrm{Si}$ & Si \\
\hline Depuración de código & Si & $\mathrm{Si}$ & $\mathrm{Si}$ & $\mathrm{Si}$ \\
\hline Detección de errores & $5 i$ & $\mathrm{Si}$ & $\mathrm{Si}$ & Si \\
\hline Refactorización & Si & Si & $\cdots$ & Si \\
\hline Diseñador Drag and Drop & $\mathrm{Si}$ & Si & Si & Si \\
\hline Versión & $\begin{array}{c}\text { Eclipse Juno } \\
4.2\end{array}$ & $\begin{array}{c}\text { Versión Beta } \\
0.2\end{array}$ & Versión Beta & $\begin{array}{l}\text { NetBeans } \\
\text { IDE } 7.4\end{array}$ \\
\hline Multiplataforma & $\begin{array}{l}\text { Windows } \\
\text { Linux } \\
\text { Mac }\end{array}$ & $\begin{array}{l}\text { Windows } \\
\text { Linux } \\
\text { Mac }\end{array}$ & $\begin{array}{l}\text { Windows } \\
\text { Linux } \\
\text { Mac }\end{array}$ & $\begin{array}{l}\text { Windows } \\
\text { Linux } \\
\text { Mac }\end{array}$ \\
\hline Open Source & Si & Si & $\mathrm{Si}$ & $\mathrm{Si}$ \\
\hline Soporte & $\begin{array}{l}\text { Google ofrece } \\
\text { un plugin ADT }\end{array}$ & Google & $\begin{array}{l}\text { MIT App } \\
\text { Inventor }\end{array}$ & .. \\
\hline Precio & Gratuito & Gratuito & Gratuito & Gratuito \\
\hline
\end{tabular}

El asistente de código es una funcionalidad que permite contar con una serie de propuestas de código para completar lo que se está invocando (Mednieks, Dornin, Meike, \& Nakamura, 2011, pág. 120). Tanto Eclipse, como Android Studio y NetBeans cuentan con asistente de código. Otra funcionalidad es la revisión de sintaxis en tiempo real, esta característica indica que el código no coincide con las reglas de sintaxis y lo subraya en tiempo real. Tanto Eclipse, como Android Studio y NetBeans cuentan con esta característica.

La depuración de código o Debugging permite ejecutar un programa de forma interactiva mientras se ve el código fuente y las variables durante la ejecución (Vogel, 2013). Tanto Eclip-
Refactorización es el proceso de reestructuración del código sin cambiar su comportamiento. Tanto Eclipse, como Android Studio y NetBeans cuentan con asistente de código. Multiplataforma consiste en la característica que tiene la IDE para poder ejecutarla en varios sistemas operativos. Adicionalmente la funcionalidad Drag and Drop consiste en tomar un elemento, arrastrarlo y colocarlo dentro de un layout en la posición que se desee.

Todas las IDEs mencionados anteriormente son Open Source, por lo que pueden ser empleadas para desarrollar aplicaciones para el sistema operativo Android de manera gratuita. De la misma manera, esto contribuye a que no se tenga que pagar un precio por licencia de desarrollo.

Android Studio es el nuevo IDE para desarrollar aplicaciones Android. Fue presentado en la conferencia Google I/O 2013. Personal de Google indicó que por ahora Android Studio está disponible en su versión 0.2 como preview, por lo que presenta algunos fallos y muchas funciones que aún faltan por implementar (aún no están disponibles), agradecieron las futuras retroalimentaciones acer- 
ca del uso de esta IDE. Está basado en Intellij IDEA. Un IDE similar a Eclipse+Plugin ADT. Según Google, Android Studio es una IDE que permitirá a los desarrolladores ser más rápidos y productivos.

Como se puede observar en la tabla, las diferencias entre los entornos de desarrollo integrado estudiados son mínimas y a pesar de que Android Studio es la IDE oficial de Google para desarrollar aplicaciones Android, se ha decidido que para la realización de este trabajo se utilizará Eclipse por ser una IDE con más trayectoria que ha demostrado ser estable y funcional, además de que cuenta con el soporte oficial de Google. Como conclusión se seleccionó Eclipse.

\section{Selección del microcontrolador}

El PIC18F25K20 es un potente microcontrolador de 28 pines que cumple con los requerimientos del microcontrolador del diagrama de bloques. Una de las ventajas del microcontrolador PIC18F25K20 es que cuenta con una IDE propietaria para programarlo: AMICUS18. Este microcontrolador también puede ser programado utilizando el programador universal PICkit 2.

\section{Requisitos funcionales}

La selección de criterios de diseño consiste en el análisis de requisitos funcionales a tener en consideración para diseñar el prototipo. De acuerdo a las entrevistas con el personal de la FLOM se definieron los requisitos funcionales que se muestran en la Figura 2.

Todas las funcionalidades mencionadas en la Figura 2 serán ejecutadas desde la aplicación Android, y se enviarán al módulo Bluetooth. El módulo Bluetooth puede ser configurado con una clave de autenticación, a su vez esta clave debe ir programada en el código de la aplicación para que se establezca la comunicación entre el dispositivo móvil y el módulo Bluetooth.

\section{Figura \# 2}

Diagrama de caso de uso.

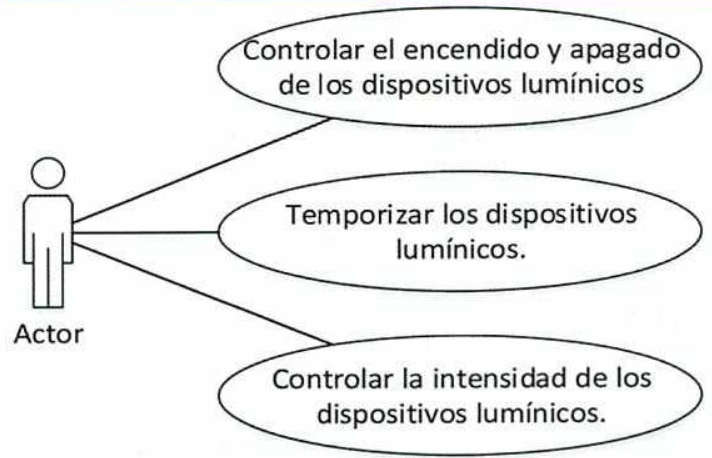

Fuente: Elaboración propia.

\section{Diseño del prototipo de control de lámparas}

El diseño del control de lámparas utilizará: 1. una aplicación para dispositivos móviles con sistema operativo Android que servirá de interfaz gráfica para el usuario; 2 . un módulo inalámbrico Bluetooth HC06 para lograr la comunicación de datos entre un dispositivo móvil y el microcontrolador; y 3 . un microcontrolador PIC18F25K20 que gestionará todas las instrucciones enviadas desde la aplicación Android. Para conocer las entradas/salidas digitales/analógicas que se utilizarán en el microntrolador PIC18F45K20 es necesario conocer la distribución de las lámparas que serán conectadas.

\section{Distribución física de las lámparas}

Las lámparas como fluorescentes se encuentran distribuidos en el escenario y en el centro del auditorio, mientras que los dimmers se encuentran en los lados. La distribución de las lámparas se aprecia en la Figura 3.

Para una mejor comprensión de las conexiones que existen entre las diferentes lámparas se los ha agrupado por letras A, B, C, D, E, F. En donde: A corresponde a las 7 lámparas del escenario; $\mathrm{B}$ corresponde a las 5 lámparas del escenario; $\mathrm{C}$ corresponde a las 60 fluorescentes del centro, este grupo ha sido dividido en 5 subgrupos C1, C2, C3, C4, C5, cada subgrupo con 12 fluorescentes; D 


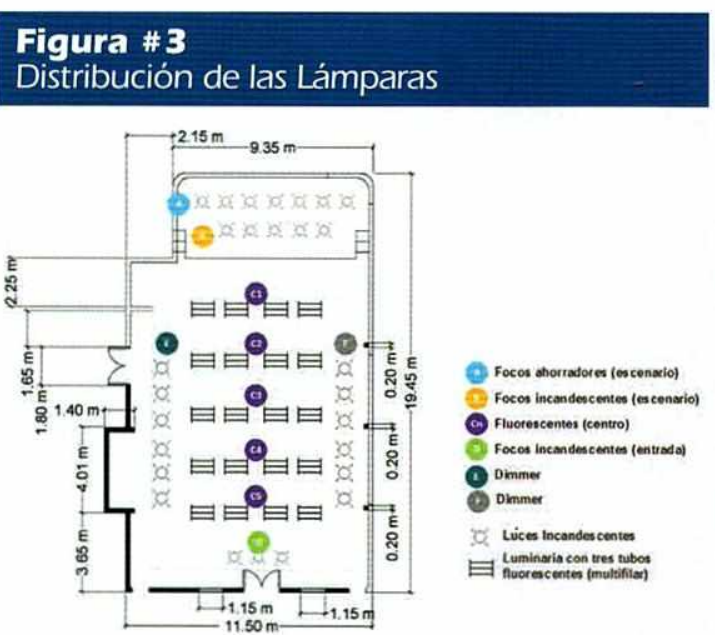

Fuente: Elaboración propia.

corresponde a las 3 lámparas de la entrada. En la Figura 4 se muestra de forma general el funcionamiento del prototipo de control de lámparas.

\section{Figura \#4}

Esquema de funcionamiento del prototipo de lámparas.

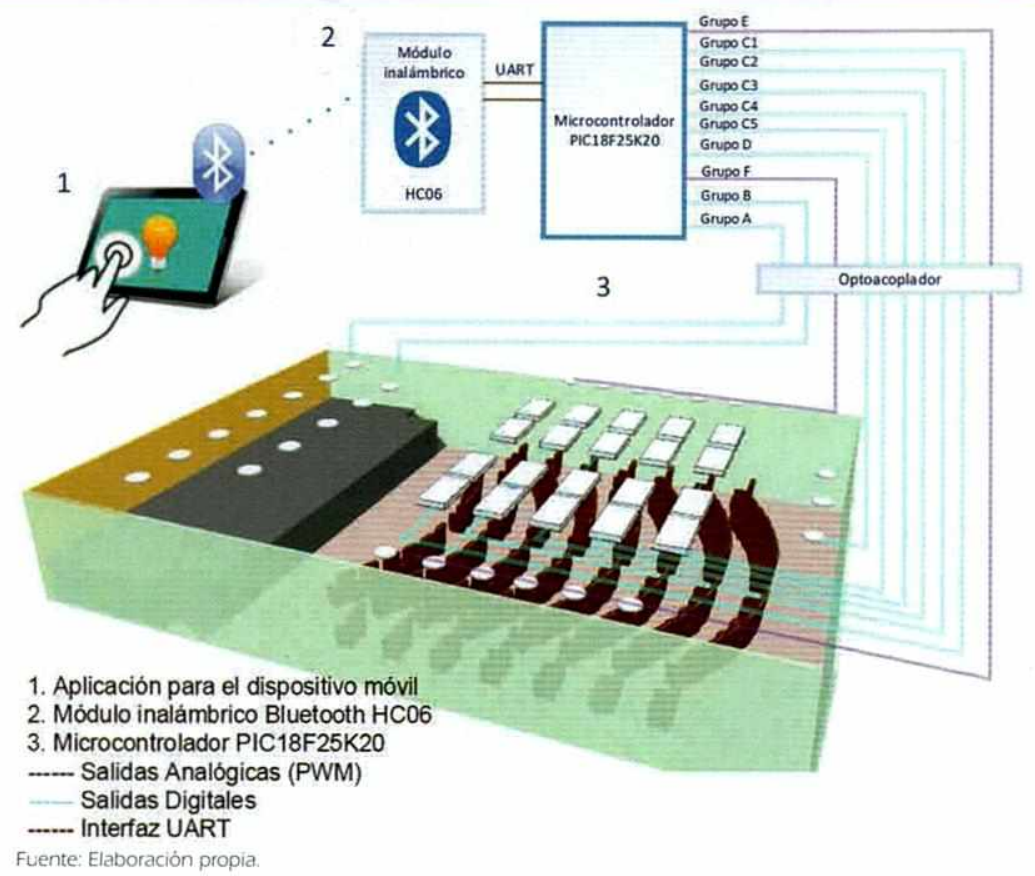

IDE Eclipse Juno. Para brindar facilidad al momento de controlar las lámparas, la aplicación ha sido diseñada en base a una pantalla de 10 pulgadas, la misma que tiene una resolución de 1280x800 px. Adicionalmente la aplicación está configurada para ejecutarse en orientación "landscape", de manera que se pueda aprovechar el mayor ancho posible de la pantalla.

\section{Maquetación de la interfaz gráfica de la aplicación}

De acuerdo a la Guía API de Android, Interfaz de usuario es todo lo que el usuario puede ver e interactuar (Android Developers). Por lo tanto la interfaz de usuario es la interfaz gráfica de la aplicación. Para la interfaz gráfica de la aplicación se hará uso de una imagen de fondo dejando atrás los tradicionales menús. Además teniendo en cuenta que la cantidad de lámparas es considerable se hace factible acceder a ellos desde una sola ventana, siendo la imagen del auditorio un referente que facilita al usuario el uso de la aplicación. El resultado final se muestra en la Figura 5.

\section{Diseño de la aplicación para disposi-} tivos móviles con sistema operativo Android

La aplicación es la herramienta que utilizará el usuario para poder controlar las lámparas del auditorio. La aplicación se instalará en un dispositivo móvil con sistema operativo Android, la cual se desarrollará con la 


\section{Figura \#5}

Diseño de la imagen de fondo del auditorio de la FLOM.

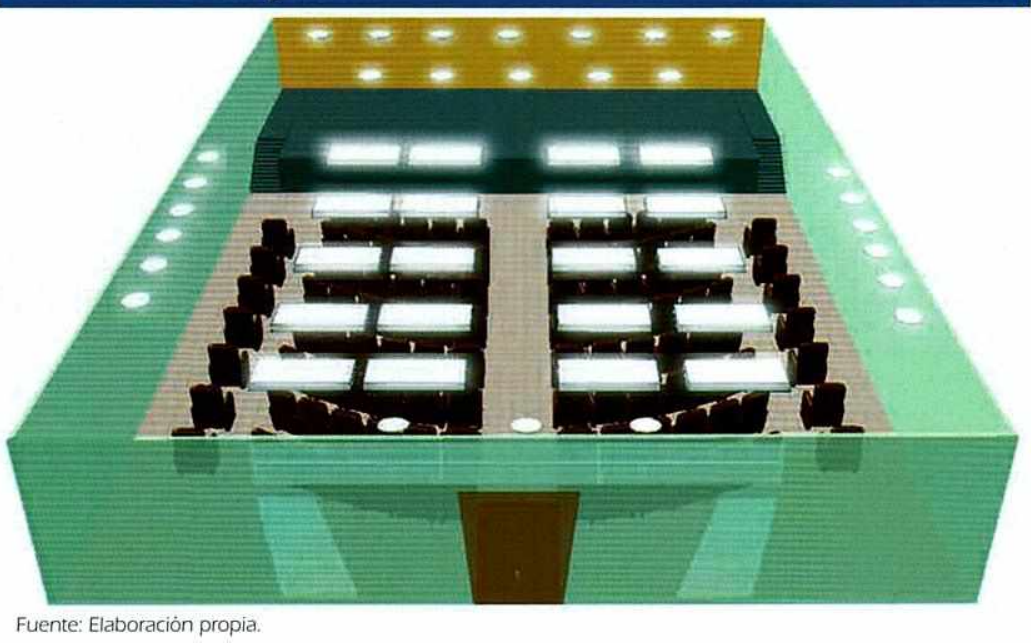

Diseño de esquema electrónico para control vía módulo inalámbrico Bluetooth

La parte electrónica en este trabajo comprende: el diseño del circuito, la simulación del módulo inalámbrico Bluetooth $\mathrm{HCO6}$ y la programación del microcontrolador PIC18F25K20.

\section{Diseño del circuito electrónico}

Para el diseño y simulación del circuito electrónico se utiliza la Suite de Diseño Proteus. Los componentes principales utilizados en el diseño del circuito electrónico son: el microcontrolador PIC18F25K20 y el módulo inalámbrico Bluetooth HC06. El microcontrolador PIC18F25K20 se encuentra entre los dispositivos que ofrecen las librerías de Proteus así que se lo asignará fácilmente. Por otro lado al no encontrarse el módulo inalámbrico Bluetooth $\mathrm{HCO}$ dentro de las librerías de Proteus, se utiliza el componente Virtual Terminal. Otros componentes adicionales que se utilizan son: resistencias, optoacopladores, fuentes de voltaje.

\section{Diseño del programa del microcon- trolador PIC18F25K20}

Para el desarrollo del programa del microcontrolador PIC18F25K20, se utilizó la IDE Amicus18 que tiene una interfaz ami- gable. Para establecer comunicación serial entre el Virtual Terminal (Módulo inalámbrico Bluetooth HC06) y el microcontrolador PIC18F25K20 es necesario definir en el código del PIC la tasa de baudios Declare Hserial_Baud $=9600$. De no incluir la tasa de baudios el valor predeterminado es de 2400 baudios. Otro valor que se puede ajustar es la paridad par o impar definiendo. El valor predeterminado es $8 \mathrm{~N} 1$ el cual indica el formato de datos: 8 bits de datos, sin bit de paridad y 1 bit de parada.

Después se definen las variables. Se requiere de una variable tipo string la cual va almacenar los datos transmitidos desde el Virtual Terminal (Módulo inalámbrico Bluetooth $\mathrm{HC} 06$ ) así que se crea una variable tipo string con capacidad de 7 caracteres, ya que ese es el número máximo de caracteres que se enviará al PIC desde la aplicación.

Los Modificadores Serout son claves en la comunicación ya que permiten transmitir y recibir datos. Con el modificador HSerIn se controla sí llega una instrucción desde el Virtual terminal, sino se dispara un salto a seguir esperando, y se repite el proceso. $\mathrm{Si}$ una instrucción es enviada se almacena en una variable.

\section{Códigos de operación}

Para lograr el control de las lámparas se ha definido un código de operación, el cual se transmitirá desde la aplicación al módulo inalámbrico Bluetooth y este último se comunicará con el microcontrolador PIC18F25K20. 
El código de operación para "controlar el encendido y apagado de las lámparas" emplea 3 dígitos. En donde el primer dígito identifica la función a realizar, en este caso encender y apagar. El segundo y el tercer dígito conforman el identificador de puerto el cual indica que puerto del microcontrolador PIC18F25K20 controla.

El código de operación para "temporizar las lámparas" emplea 3 dígitos y funciona de igual forma que el código anterior, a diferencia que la temporización es llevada a cabo por la aplicación para el dispositivo móvil. El máximo tiempo de temporización que se puede configurar es de 3599 segundos (59:59).

El código de operación para “controlar la intensidad de las lámparas" emplea 4 dígitos. En donde el primer dígito indica la función a realizar, en este caso controlar la intensidad lumínica. El segundo, tercero y cuarto dígito indican la variación de intensidad lumínica, entre un valor de 0 a 255 .

\section{Simulación del circuito electrónico}

De igual forma como se utiliza Proteus para el diseño, así mismo se lo utiliza para la simulación. El "virtual terminal" es el componente de Proteus que en este caso funciona como la interfaz de usuario para controlar las lámparas a través de los códigos de operaciones definidos anteriormente.

Para empezar, el código del PIC, luego de guardado y compilado desde la IDE Amicus18, debe ser programado en el microcontrolador. Posteriormente para realizar la simulación se requiere enviar a un código de operación. En este caso se envía el código 109 para encender el grupo C3, en respuesta el Virtual Terminal muestra un mensaje de "Código Recibido: 109 Grupo C3 Encendido", tal como se muestra en la Figura 6.

\section{Implementación y pruebas del proto-} tipo de control de lámparas

Para la implementación del prototipo de control de las lámparas se procedió a instalar la aplicación en un dispositivo móvil. Posteriormente se realizó el armado del circuito en un protoboard, y finalmente, se procedió a ejecutar las pruebas y verificaciones del funcionamiento.

\section{Instalación de la aplicación Android}

Para el proceso de instalación se escogió como dispositivo de pruebas una tablet marca Samsung Galaxy Tab2 10.1 modelo GT-P5100, con sistema operativo Android versión 4.1.2, resolución de $1280 \mathrm{x} 800 \mathrm{y}$ Bluetooth v. 3.0. En la Figura 7 se muestra la aplicación instalada.

En la Figura 8 se muestra las funcionalidades que se pueden realizar desde la aplicación. 


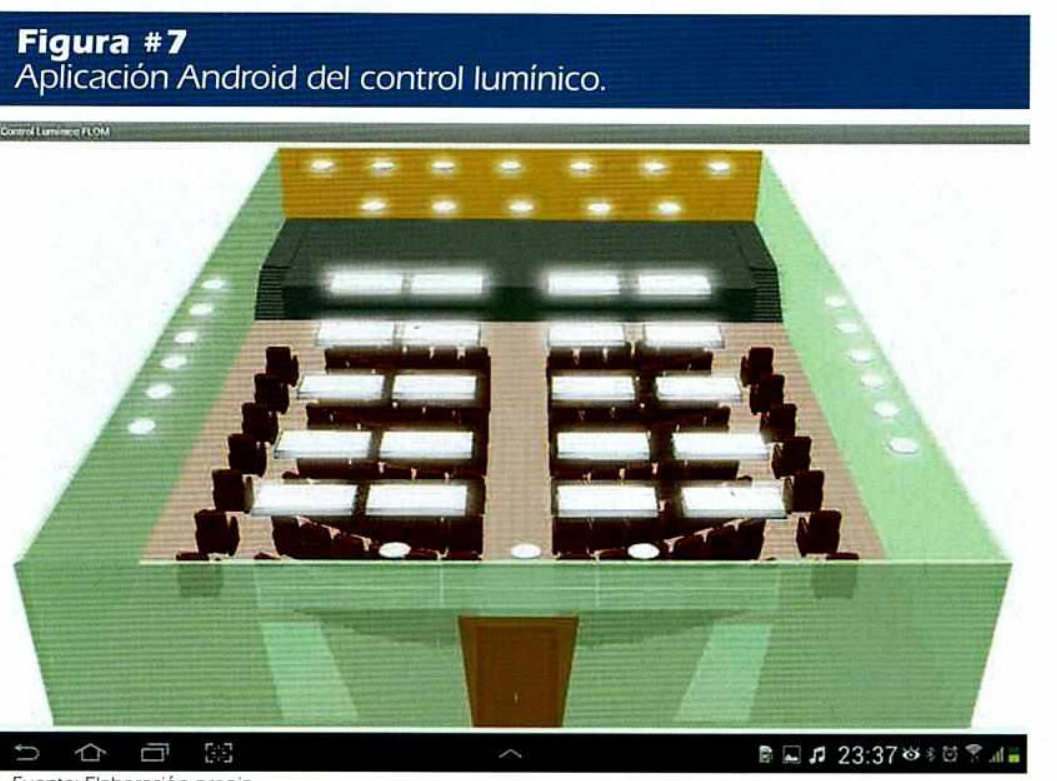

Fuente: Elaboración propia.
Implementación del circuito electrónico

Se procedió armar en el protoboard el circuito electrónico. Se realizó las conexiones de manera que se pudiera establecer la comunicación USART respectiva entre el módulo inalámbrico Bluetooth HC06 y el microcontrolador PIC18F25K20. Posteriormente para simular la carga fueron conectadas todas las salidas a LEDs.
Figura \#8

Guía de las funcionalidades del prototipo de control de las lámparas.

Encender o apagar un dispositivo lumínico Para encender o apagar presione sobre el grupo respectivo de los dispositivos lumínicos.

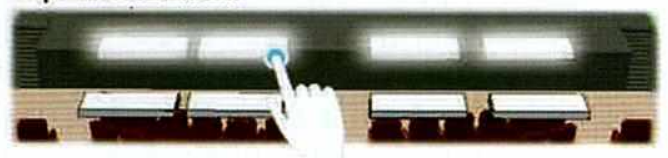

Temporizar los dispositivos luminicos

1. Para temporizar mantenga presionado por un segundo el grupo respectivo de los dispositivos lumínicos.

2. Espera a que se abra el cuadro de diálogo y especifique el tiempo.

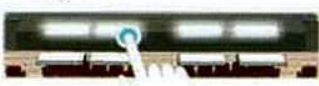

$2=\tan$

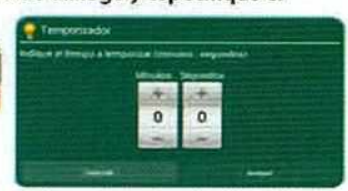

Controlar la intensidad luminica

1. Para controlar la intensidad lumínica mantenga presionado el grupo respectivo de los dispositivos lumínicos.

gruporespectivo izquierda a derecha para aumentar la intensidad o vicerversa para disminuirla.

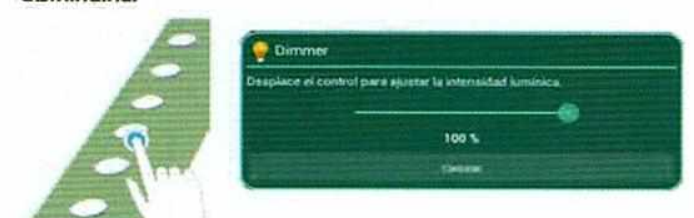

\section{Menú}

Para encender o apagar todos los dispositivos lumínicos seleccione la opción "Encender y apagar"

Para controlar la intensidad lumínica de los dos juegos de dimmers seleccione la opción "Dimmers".

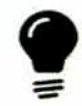

Para visualizar el estado de temporizado de los dispositivos lumínicos seleccione la opción "Estados" También desde esta opción se puede cancelar una temporización seleccionando el botón "Cancelar".

Fuente: Elaboración propia.
Prueba de funcionamiento del prototipo de control de lámparas

Para las pruebas de funcionamiento se implementó el prototipo en una maqueta del auditorio y se elaboró un plan de pruebas. El plan de pruebas consistió en ir probando cada una de las funcionalidades que ofrece el prototipo de control de lámparas. Las pruebas que se realizaron fueron de: conexión, encendido/apagado, temporización e intensidad lumínica.

\section{Prueba de conexión}

El objetivo de esta prueba es comprobar la comunicación establecida entre el dispositivo móvil y el módulo inalámbrico Bluetooth. Las herramientas empleadas son: el dispositivo móvil con la aplicación instalada y el control de lámparas.

El procedimiento realizado consiste en: 1.Verificar que las conexiones respectivas estén en funcionamiento, 2. Iniciar la aplicación desde el dispositivo móvil y 3 . Observar el mensaje de bienvenida generado en la aplicación. En la Tabla 3 se muestra el resultado. 


\section{Tabla \#3}

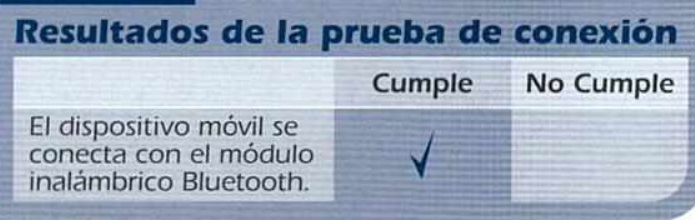

Fuente: Elaboración propia.

Prueba de "encendido y apagado de las lámparas"

El objetivo de esta prueba es comprobar el encendido y apagado de cada uno de las lámparas. En la pantalla principal de la aplicación, se verificó el encendido y apagado de cada uno de los grupos A, B, C1, C2, C3, C4, C5 y D, esto se lo realizó mediante un gesto táctil sobre cada grupo de acuerdo a la distribución de las lámparas en la interfaz gráfica de la aplicación.

Los lámparas que son controladas con dimmer, grupo $\mathrm{E}$ y $\mathrm{F}$, también pueden ser llevadas al valor mínimo y máximo de intensidad mediante un gesto táctil de manera similar a los demás grupos, esta funcionalidad adicional es gracias al prototipo. En la Tabla 4 se muestra el resultado.

\section{Tabla \#4}

Resultados de la prueba de encendido y apagado de I

\begin{tabular}{|c|c|c|}
\hline $\begin{array}{l}\text { Prueba de Encendido } \\
\text { y Apagado }\end{array}$ & Cumple & No Cumple \\
\hline Grupo A & $\gamma$ & \\
\hline Grupo B & $\frac{\gamma}{\gamma}$ & \\
\hline Grupo Cl & $\bar{\gamma}$ & \\
\hline Grupo C2 & j & \\
\hline Grupo C3 & j & \\
\hline Grupo C4 & $\gamma$ & \\
\hline Grupo C5 & $\checkmark$ & \\
\hline Grupo D & $\checkmark$ & \\
\hline Grupo E & $\gamma$ & \\
\hline Grupo F & $\checkmark$ & \\
\hline
\end{tabular}

Fuente: Elaboración propia

\section{Prueba de "temporizar las lámparas"}

En la pantalla principal de la aplicación, se verificó la temporización de cada uno de los grupos, esto se lo realizó manteniendo presionado por más de un segundo sobre cada grupo de acuerdo a la distribución de las lámparas en la interfaz gráfica, se configuró el tiempo de 10 segundos y se comparó con un reloj online para comprobar el tiempo transcurrido. En la Tabla 5 se muestra el resultado.

\begin{tabular}{|c|c|c|}
\hline \multicolumn{3}{|c|}{$\begin{array}{l}\text { Resultados de la prueba de } \\
\text { temporización de las lámparas }\end{array}$} \\
\hline Prueba de Temporizar & Cumple & No Cumple \\
\hline Grupo A & $\checkmark$ & \\
\hline Grupo B & $\sqrt{ }$ & \\
\hline Grupo Cl & $\gamma$ & \\
\hline Grupo C2 & $\gamma$ & \\
\hline Grupo C3 & r & \\
\hline Grupo C4 & $\gamma$ & \\
\hline Grupo C5 & $\gamma$ & \\
\hline Grupo D & $\gamma$ & \\
\hline
\end{tabular}

Prueba de "control de la intensidad de las lámparas"

En la pantalla principal de la aplicación, se verificó el control de la intensidad del Grupo E y F, esto se lo realizó manteniendo presionado por más de un segundo sobre cada grupo de las lámparas en la interfaz gráfica y se estableció la intensidad lumínica utilizando la barra deslizadora. En la Tabla 6 se encuentra la fuente de referencia se muestra el resultado.

\begin{tabular}{l} 
Tabla \#6 \\
$\begin{array}{l}\text { Resultados de la prueba de control } \\
\text { de la intensidad de las lamparas }\end{array}$ \\
$\begin{array}{l}\text { Prueba de Temporizar Cumple No Cumple } \\
\text { Grupo E }\end{array}$ \\
\hline Grupo F \\
\hline
\end{tabular}

Fuente: Elaboración propia

\section{Tiempo de respuesta del prototipo de control de lámparas}

La manera convencional de encender y apagar las lámparas en el auditorio tiene un tiempo que puede variar en función de la velocidad y la distancia. Suponiendo que un individuo se encuentra en el pódium del 
escenario y debe recorrer 19.45 metros de distancia para llegar al otro extremo del auditorio para encender o apagar la/las lámparas, el tiempo que tomaría se puede deducir de una regla de 3 compuesta, como se muestra a continuación:

\begin{tabular}{|c|c|c|c|c|}
\hline 8 segundos & $\rightarrow$ & 10 pasos & $\rightarrow$ & 4 metros \\
\hline Tiempo & $\rightarrow$ & \# pasos & $\rightarrow$ & 19.45 metros \\
\hline
\end{tabular}

$$
\begin{aligned}
& \# \text { pasos }=\frac{(19.45 \text { metros } * 10 \text { pasos })}{4 \text { metros }}=49 \text { pasos } \\
& \text { Tiempo }=\frac{(49 \text { pasos } * 8 \text { segundos })}{10 \text { pasos }}=39.2 \text { segundos }
\end{aligned}
$$

Por lo tanto, la forma convencional de encender y apagar un dispositivo lumínico en el auditorio toma un tiempo de 39.2 segundos. El tiempo obtenido es el valor "ideal" asumiendo que el individuo al llegar podrá escoger de manera rápida el interruptor deseado. Mientras que con el prototipo el encendido/ apagado de un dispositivo lumínico toma un tiempo menor a 2 segundos. En la Tabla 7 se muestra los resultados de las pruebas del tiempo empleado para encender un grupo de lámparas.

\section{Tabla \# 7 \\ Pruebas de tiempo de encendido en 19.45 metros de forma convencional y desde la aplicación \\ De forma Desde la convencional aplicación

\begin{tabular}{lcc} 
Tiempo de Encendido & 39.2 & $\leq 2$ \\
en $19.45 \mathrm{~m}$ & segundos & segundos \\
\hline
\end{tabular} \\ Fuente: Elaboración propia.}

Por otro lado asumiendo que cada dispositivo lumínico toma un segundo en encender/apagar, entonces encender/apagar todos las lámparas uno por uno toma un tiempo aproximado de 8 segundos. Mientras que con el prototipo el encendido/apagado de todos las lámparas se logra en un tiempo menor a 2 segundos. En la Tabla 8 se muestra el tiempo empleado para encender todos las lámparas de forma convencional y desde la aplicación.

\section{Tabla \#8}

Pruebas de tiempo de encendido de

todas las lámparas de forma

convencional y desde la aplicación

\begin{tabular}{|ccc}
\hline & $\begin{array}{c}\text { Deforma } \\
\text { convencional }\end{array}$ & $\begin{array}{c}\text { Desde la } \\
\text { aplicación }\end{array}$ \\
\hline Tiempo de Encendido & 8 segundos & $\leq 2$ \\
de todas los lámparas & & segundos \\
\hline
\end{tabular}

Fuente: Elaboración propia.

Las pruebas de tiempo de respuesta realizadas en el prototipo de control de lámparas han logrado cumplir las expectativas deseadas. El tiempo de respuesta del prototipo es mínimo garantizando que todas las acciones tomadas desde la aplicación sean rápidas, logrando así optimizar el tiempo de control dentro del auditorio.

\section{Conclusiones}

Luego de la comparación de los módulos inalámbricos Bluetooth RN41 y HC06, se dio paso a la selección del módulo inalámbrico Bluetooth HCO6 resultando ser suficiente para efectos de diseño del prototipo.

Los casos de uso UML fueron definidos gracias al análisis de los requisitos funcionales obtenidos a través del personal de la FLOM lo cual permitió lograr el desarrollo de la aplicación móvil Android a nivel de funcionalidades.

En base a las librerías, recursos necesarios, disponibilidad de ayuda y soporte de Google hacia la IDE Eclipse por consiguiente éstas fueron características que hicieron que la IDE facilitara el proceso de desarrollo y escritura de código de la aplicación móvil Android.

El diseño del circuito electrónico fue realizado en Proteus y gracias al componente Virtual Terminal se logró realizar la simulación del módulo inalámbrico Bluetooth en conjunto con el microcontrolador.

La simulación realizada en Proteus per- 
mitió comprobar el envío y recepción de los códigos de operaciones establecidos demostrando de esta forma su funcionalidad de encendido/apagado, temporización y control de intensidad lumínica.

El uso de una tablet de 10 pulgadas durante el desarrollo y pruebas de la aplicación para el dispositivo móvil Android, demostró ser una gran opción, dando como resultado una mejor experiencia en la interfaz de usuario garantizando un buen uso y facilidad de control.

El conjunto de herramientas de hardware y software empleados en el desarrollo del prototipo demostraron ser los adecuados para el diseño e implementación del mismo, por lo tanto se puede afirmar que en base a los resultados obtenidos Android y Bluetooth forman una gran solución para el control.

Luego de haber incorporado todas las funcionalidades, se logró determinar que el prototipo ofrece una mejora en el control de las lámparas ya que otorga nuevas funcionalidades que no se pueden realizar con el accionamiento manual.

Con el prototipo, el tiempo empleado para controlar el encendido y apagado es menor a 2 segundos, es decir, el proceso del control de las lámparas de la FLOM ha reflejado una mejora en el tiempo de respuesta.

\section{Bibliografia}

Android Developers. (2013). Android Developers. Retrieved from http://developer. android.com/tools/revisions/platforms. html

Android Developers. (2013). Android Developers. Retrieved from http://developer. android.com/sdk/installing/studio.html
Asociación Española de Domótica. (2013). CEDOM. Retrieved from http://wnww. cedom.es/sobre-domotica/que-es-domotica

Bluetooth SIG. (2013). Blietooth. Retrieved from http://www.bluetooth.com/Pages/ using-bluetooth-products.aspx

Bluetooth SIG. (2013). Blwetooth Developer Portal. Retrieved from http://developer. bluetooth.org/Pages/default.aspx

Carballar, J. (2010). WVi-Fi : to que se necesita conocer: Madrid.

Gironés, T. (2012). El gran libro de Android. Barcelona: MARCOMBO, S.A. .

Guangzhou HC Information Technology' Co.,Ltd. . (2013). SLABPROG. Retrieved from http://silabs.org.ua/bc4/hc06.pdf

Huidobro, J. (2007). Sistemas telemáticos (Tercera ed.). Madrid: Paraninfo.

Huidobro, J. (2011). Radiocommicaciones. Creaciones Copyright.

IDC. (2014). IDC. Retrieved from wwwidc. com/prodserv/smartphone-os-marketshare.jsp

Jamsrich Parsons, J., \& Oja, D. (2008). Conceptos de Computación: Nuevas Perspectivas. Cengage Learning.

Jatschka, T., \& Tschofen, R. (2004). Ethernet and Wireless/Mobile Network Technologies. In R. Zurawski, The Industrial Information Teclnology' Handbook.

Kendall, K., \& Kendall, J. (1997). Análisis $y^{\prime}$ Diseño de sistemas (Tercera ed.). 
Lee, W.-M. (2011). Beginning Android 4 Application Development. Indianápolis: Wiley Publishing, Inc.

Martín, J. (2009). Instalaciones domóticas. Editex.

Mednieks, Z., Dornin, L., Meike, B., \& Nakamura, M. (2011). Programming Android. O'Reilly Media. Retrieved from http:// books.google.es/books?id=bKo_1uED7 $2 \mathrm{EC} \& \mathrm{dq}=$ code + assistant $+\mathrm{ide} \& \mathrm{hl}=\mathrm{es} \& \mathrm{~s}$ ource $=$ gbs_navlinks_s

Microchip. (2013). Microcbip Technology Inc. Retrieved from http://ww1.microchip. com/downloads/en/DeviceDoc/rn-41 ds-v3.42r.pdf

Millennial Media. (2013). Mobile Mix the mobile device index. Retrieved from www.millennialmedia.com/mobile-intelligence/ mobile-mix

Molisch, A. (2010). Wireless Communications (Segunda ed.). Wiley.

NetBeans. (2012). NetBeans. Retrieved from http://plugins.netbeans.org/plu$\operatorname{gin} / 19545 /$

Nielsen, J., \& Budiu, R. (2013). Usabilidad en dispositivos móviles. Anaya Multimedia.

Noble, J. (2012). Programming Interactivity (Segunda ed.). Estados Unidos: O'Reilly Media.

Object Technology International, Inc. (2003, Febrero s/d). Eclipse. Retrieved from http://www.eclipse.org/whitepapers/ eclipse-overview.pdf

Pal, A. (2011). Microcontrollers: Principles and Applications. New Delhi: PHI.
Scarfone, K., \& Padgette, J. (2012). Gride to Bluetooth Security. Estados Unidos: National Institute of Standars and Technology. Retrieved from http://csrc.nist.gov/ publications/nistpubs/800-121-rev1/ sp800-121_rev1.pdf

Sequoia-Technology. (S/A). Squidoo. Retrieved from An easy guide to Bluetooth Modules: http://www.squidoo.com/ bluetooth-modules

Shneiderman, B. (1998). Designing the User Interface.

TazTag. (n.d.). TazTag Secure Mobile Appliances. Retrieved from http:// taztag.com/index.php?option $=\mathrm{com}_{-}$ content\&view $=$ article\&id $=104$

Thompson, T., Kumar, B., \& Kline, P. (2008). Bhetooth Application Programming with the Java APIs.

Tomás, J. (2013). El gran libro de Android (Tercera ed.). Barcelona: Marcombo.

Tomasi, W. (2003). Sistemas de Comunicaciones Electrónicas (Cuarta ed.). México: Prentice Hall.

Umar, A. (2004). Mobile Computing and Wireless Communications.

Verón, J. (2009). Prácticas de Redes.

Vogel, L. (2013, Enero 24). Vogella. Retrieved from http://www.vogella.com/ articles/EclipseDebugging/article. html\#resources

Wolber, D., Abelson, H., Spertus, E., \& Looney, L. (2011). App Inventor:

Zahariadis, T. (2003). Home Networking Technologies and Standards. 
Ma. Daniela Ronquillo Pilamunga

Ingeniería en Telecomunicaciones de la Universidad Espíritu Santo - Ecuador.

E-mail: mronqui@uees.edu.ec

\section{César Martin Moreno}

Magister en Ciencias en Ingeniería Eléctrica de la Escuela Superior Politécnica Del Litoral (ESPOL)

Universidad Espiritu Santo - Ecuador

E-mail: cesarmartin@uees.edu.ec 
